\title{
Scalable Image Retrieval Systems and Applications
}

\author{
Karan Ravindran Pillay, Omkar Upendra Khadilkar
}

Department of Computer Technology, Pune.

\begin{abstract}
:
Advances in information storage and image acquisition technologies have enabled the creation of enormous image datasets. during this situation, it's necessary to develop applicable data systems to with efficiency manage these collections. the most typical approaches use the supposed Content-Based Image Retrieval (CBIR) systems. Basically, these systems attempt to retrieve pictures like a user-defined specification or pattern (e.g., form sketch, image example). Their goal is to support image retrieval supported content properties (e.g., shape, color, texture), typically encoded into feature vectors. one among the most benefits of the CBIR approach is that the chance of AN automatic retrieval method, rather than the standard keywordbased approach, thattypically needs terribly toilsome and long previous annotation of info pictures. The CBIR technology has been utilized in many applications like fingerprint identification, variety data systems, digital libraries, crime bar, medicine, historical analysis, among other
\end{abstract}

\section{Introduction}

Advances in knowledge storage and image acquisition technologies have enabled the creation of enormousimage datasets. so as to traumatize these knowledge, it's necessary to develop applicable info systems to with efficiency manage these collections. Image looking out is one among the foremost necessary services that require to be supported by such systems. In general, 2 totally different approaches are applied to permit looking out on image collections: one supported image matter medatada and another supported image content info. the primary retrieval approach is predicated on attaching matter information to every image and uses ancient information question techniques to retrieve them by keywords. but these systems need a previous annotation of the information pictures, that could be a terribly toilsome and long task. moreover, the annotation method is sometimes inefficient as a result of users, generally, don't create the annotation in a very systematic means. In fact, different users tend to use different words to explain a same image characteristic. the dearth of systematisation within the annotation method decreases the performance of the keywordbased image search.

\section{Literature Survey}

Paper Name: Relevance Feedback: A Power Tool for Interactive Content-Based Image Retrieval Author: Yong Rui, Thomas S. Huang Description: Content-based image retrieval (CBIR) has become one of the most active research areas in the past few years. Many visual feature representations have been explored and many systems built. While these research efforts establish the basis of CBIR, the usefulness of the proposed approaches is limited. Specifically, these efforts have relatively ignored two distinct characteristics of CBIR systems: 1) the gap between high-level concepts and low-level features, and 2) subjectivity of human perception of visual content. This paper proposes a relevance feedback based interactive retrieval approach, which effectively takes into account the above two characteristics in CBIR. During the retrieval process, the user's high-level query and perception subjectivity are captured by dynamically updated weights based on 
the user's feedback. The experimental results over more than 70000 images show that the proposed approach greatly reduces the user's effort of composing a query, and captures the user's information need more precisely.

Paper Name: Semantics-Preserving Hashing for Cross-View Retrieval

Author : Zijia Lin, Guiguang Ding, Mingqing Hu

Description : With benefits of low storage costs and high query speeds, hashing methods are widely researched for efficiently retrieving large-scale data, which commonly contains multiple views, e.g. a news report with images, videos and texts. In this paper, we study the problem of cross-view retrieval and propose an effective Semantics-Preserving Hashing method, termed SePH. Given semantic affinities of training data as supervised information, SePH transforms them into a probability distribution and approximates it with tobe-learnt hash codes in Hamming space via minimizing the Kullback-Leibler divergence

Paper Name: Content-Based Image Retrieval at the End of the Early Years

Author: Arnold W.M. Smeulders, Marcel Worring

Description: The paper presents a review of 200 references in content-based image retrieval. The paper starts with discussing the working conditions of content-based retrieval: patterns of use, types of pictures, the role of semantics, and the sensory gap. Subsequent sections discuss computational steps for image retrieval systems. Step one of the review is image processing for retrieval sorted by color, texture, and local geometry. Features for retrieval are discussed next, sorted by: accumulative and global features, salient points, object and shape features, signs, and structural combinations thereof. Similarity of pictures and objects in pictures is reviewed for each of the feature types, in close connection to the types and means of feedback the user of the systems is capable of giving by interaction. We briefly discuss aspects of system engineering: databases, system architecture, and evaluation. In the concluding section, we present our view on: the driving force of the field, the heritage from computer vision, the influence on computer vision, the role of similarity and of interaction, the need for databases, the problem of evaluation, and the role of the semantic gap

\section{Existing System}

To increase and ubiquitous accessibility of visual data on the Web have led to the prosperity of research activity in image search or retrieval. With the unawareness of graphic content as a grade trace, approaches with text search organizations for visual retrieval may suffer inconsistency between the text words and visual content.

\section{Proposed System}

In proposed system, commonest approaches use the so-called Content-Based Image Retrieval (CBIR) systems. Basically, these organizations try to recover images related to a userdefined requirement or pattern (e.g., shape sketch, image example). Their goal is to support image recovery based on content properties (e.g., shape, color, texture), usually encoded into feature vectors.

\section{Conclusion}

In this system, we propose method of content-based image retrieval area. Firstly, we have obtainable a set of creates directing to define exactly the main interrelated ideas. Next, we have described the main issues that need to be taken into account when designing this kind of image retrieving system: definition of appropriate image descriptors, feature vector representation and indexing, interaction mechanisms, among others.

\section{References}


[1] Y. Rui, T. S. Huang, M. Ortega, and S. Mehrotra, "Relevance feedback: a power tool for interactive content-based image retrieval," IEEE Transactions on Circuits and Systems for Video Technology, vol. 8, no. 5, pp. 644-655, 1998

[2] Z. Lin, G. Ding, M. Hu, and J. Wang, "Semantics-preserving hashing for cross-view retrieval," in IEEE Conference on Computer Vision and Pattern Recognition (CVPR), 2015, pp. 3864-3872.

[3] W. Smeulders, M. Worring, S. Santini, A. Gupta, and R. Jain, "Content-based image retrieval at the end of the early years," IEEE Transactions on Pattern Analysis and Machine Intelligence, vol. 22, no. 12, pp. 1349-1380, 2000.

[4] X. Li, T. Uricchio, L. Ballan, M. Bertini, C. G. Snoek, and A. D. Bimbo, "Socializing the semantic gap: A comparative survey on image tag assignment, refinement, and retrieval," ACM Computing Surveys (CSUR), vol. 49, no. 1, p. 14, 2016.

[5] Z. Lin, G. Ding, M. Hu, and J. Wang, "Semantics-preserving hashing for cross-view retrieval," in IEEE Conference on Computer Vision and Pattern Recognition (CVPR), 2015, pp. 3864-3872.

[6] M. S. Lew, N. Sebe, C. Djeraba, and R. Jain, "Content-based multimedia information retrieval: State of the art and challenges," ACM Transactions on Multimedia Computing, Communications, and Applications (TOMM), vol. 2, no. 1, pp. 1-19, 2006.

[7] Y. Liu, D. Zhang, G. Lu, and W.-Y. Ma, "A survey of contentbased image retrieval with high-level semantics," Pattern Recognition, vol. 40, no. 1, pp. 262-282, 2007.

[8] D. G. Lowe, "Distinctive image features from scale invariant keypoints," International Journal of Computer Vision, vol. 60, no. 2, pp. 91-110, 2004.

[9] J. Sivic and A. Zisserman, "Video Google: A text retrieval approach to object matching in videos," in IEEE Conference on Computer Vision and Pattern Recognition, 2003, pp. 1470- 1477. 10.D. Nister and H. Stewenius, "Scalab 